\title{
A symmetry result on submanifolds of space forms and applications
}

\author{
Ali Maalaoui ${ }^{(1)} \&$ Vittorio Martino ${ }^{(2)}$
}

\begin{abstract}
In this paper we prove a symmetry result on submanifolds of codimension one in a $n+1$-dimensional space form, related to the geodesic distance function and to the normal curvature of some fixed vector field. As applications we will prove sphere characterization type theorems for Kähler manifolds endowed with a toric group action.
\end{abstract}

\section{Introduction}

In this paper we will prove a symmetry result on submanifolds of codimension one (hypersurface type) in a $n+1$-dimensional space form, related to the geodesic distance function and to the normal curvature of some vector field. We recall that a space form is by definition a complete Riemannian manifold with constant sectional curvature $K$. Let then $V:=V^{n+1}, n \geq 1$ be a smooth complete manifold of dimension $n+1$ and let $g$ be a Riemannian metric on $V$ and $\nabla$ be the related Levi-Civita connection of $g$. Let us

\footnotetext{
${ }^{1}$ Department of Mathematics, Rutgers University - Hill Center for the Mathematical Sciences 110 Frelinghuysen Rd., Piscataway 08854-8019 NJ, USA. E-mail address: maalaoui@math.rutgers . edu

${ }^{2}$ Dipartimento di Matematica, Università di Bologna, piazza di Porta S.Donato 5, 40127 Bologna, Italy. E-mail address: martino@dm.unibo.it
} 
consider $M:=M^{n}$ a smooth, compact, orientable and with no boundary, embedded manifold on $V$, of dimension $n$. We consider on $M$ the metric induced by $g$ and the related induced Levi-Civita connection. Let us call $N$ the inner unit normal to $M$. The Second Fundamental Form $h$ on $M$ is the symmetric tensor defined on $T M$, the tangent bundle of $M$, in the following way:

$$
h(X, Y)=g\left(\nabla_{X} Y, N\right), \quad \forall X, Y \in T M
$$

Definition 1.1. Let us consider a unit smooth vector field $X \in T M$. We will call the Normal Curvature of $M$ referred to the vector field $X$ the coefficient of the Second Fundamental Form related to the vector field $X$ :

$$
\mathcal{C}^{X}:=h(X, X)=g\left(\nabla_{X} X, N\right)
$$

The distance function $d$ on $V$, related to $g$, is defined as follows: let us consider any two points $p_{0}, p_{1} \in V$, then

$$
d\left(p_{0}, p_{1}\right)=\int_{t_{0}}^{t_{1}} \sqrt{g(\dot{r}(t), \dot{r}(t))} d t
$$

where

$$
r:\left[t_{0}, t_{1}\right] \rightarrow V, \quad r\left(t_{0}\right)=p_{0}, \quad r\left(t_{1}\right)=p_{1}
$$

is a minimal geodesic in $V$ connecting $p_{0}$ and $p_{1}$. For $q \in V$, if $R>0$ is such that $\exp _{q}$ (the exponential map at $q$ ) is a diffeomorphism on the ball $B(0, R) \subset T_{q} V$ then the geodesic ball $\mathcal{B}(q, R)$ of center $q$ and radius $R$ is the image set in $V$

$$
\mathcal{B}(q, R)=\exp _{q}(B(0, R))
$$

moreover if the closed ball $\overline{B(0, R)}$ is also contained in an open set $U \subset T_{q} V$ where $\exp _{q}$ is a diffeomorphism, then the geodesic sphere is $\partial \mathcal{B}(q, R)=$ $\exp _{q}(\partial B(0, R))$.

We can now state our result: 
Theorem 1.2. Let $V$ be a $n+1$-dimensional Riemannian manifold with constant sectional curvature $K$ and let $M$ be a smooth, orientable, compact, connected, with no boundary, embedded manifold on $V$, of dimension $n$. Suppose there exist a point $q \notin M$ and a non-singular vector field $X \in T M$ such that

$$
X(d(q, p))=0, \quad \forall p \in M
$$

Let $\mathcal{C}^{X}$ be the Normal Curvature of $M$ referred to the vector field $X$. We have two cases:

i) $\quad K \leq 0$. If $\mathcal{C}^{X}$ is constant on $M$, then $M$ is a geodesic sphere in $V$;

ii) $K>0$. If $M$ belong to $\mathcal{B}\left(q, \frac{\pi}{\sqrt{K}}\right)$ and $\mathcal{C}^{X}$ is constant on $M$, then $M$ is a geodesic sphere in $V$.

Remark 1.3. We recall that fixed a point $q \in V$ then a conjugate point to $q$ is a point $p \in V$ such that there is no uniqueness for minimal geodesic

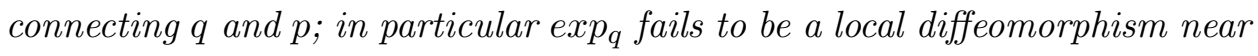
$p$. Then when $K>0$ we need to assume that $M$ is contained in the geodesic ball $\mathcal{B}\left(q, \frac{\pi}{\sqrt{K}}\right)$ in order to avoid conjugate points. In fact by a classical result we have that if $V$ has positive constant sectional curvature $K$, then the first conjugate point along any geodesic starting from $q$ occurs at distance at least $\frac{\pi}{\sqrt{K}}$. On the other hand when $K \leq 0$ then there are no conjugate points to any point of $V$.

We will give three applications of this result: the first one on almost symplectic manifolds, with the Normal Curvature referred to the hamiltonian vector field of the hypersurface $M$; the second one on Kähler manifolds endowed with a toric group action; finally we will specialize at the case of Reinhardt domains in $\mathbb{C}^{n+1}$ where we obtain as corollary the result in [11]: in that paper the second author, motivated by two recent works by Hounie 
and Lanconelli ([8], [9]), proved an Alexandrov type theorems for Reinhardt domains in $\mathbb{C}^{n+1}$ using the Characteristic Curvature rather than the Levi ones. Next we prove our result Theorem (1.2)

Proof. First of all, since the vector field $X$ is non-singular we can always normalize it such that $g(X, X)=1$. For every $p \in M$ let us consider an integral curve $\gamma$ of any vector field $Y \in T M$ passing through $p$, namely: let $\varepsilon>0$, then $\gamma:(-\varepsilon, \varepsilon) \rightarrow M$ such that

$$
\gamma(0)=p, \quad \text { and } \quad \frac{d}{d s} \gamma(s)=Y_{\gamma(s)}, \quad \forall s \in(-\varepsilon, \varepsilon)
$$

Since there are no conjugate points to $q$ on $M$, then the exponential map $\exp _{q}$ has no singularities on $M$. We consider the smooth family $r(s, t)$ of unique minimal geodesics connecting $q$ and $\gamma$, that is:

$$
\begin{gathered}
r(s, t):(-\varepsilon, \varepsilon) \times[0,1] \longrightarrow V \\
\left\{\begin{array}{l}
\frac{D}{\partial t} \frac{\partial}{\partial t} r(s, t)=\nabla_{\dot{r}} \dot{r}=0 \\
r(s, 0)=q, \quad r(s, 1)=\gamma(s)
\end{array}\right.
\end{gathered}
$$

We will always denote by a dot · the derivative with respect to $t$. Moreover, by setting

$$
\ell_{\gamma(s)}:=d(q, \gamma(s))
$$

then we have parametrized the geodesics such that they have constant speed (with respect to $\mathrm{t}$ )

$$
|\dot{r}(s, t)|=\ell_{\gamma(s)}, \quad \forall t \in[0,1]
$$

Let us define the following function defined on $M$

$$
E: M \rightarrow \mathbb{R}, \quad E(p):=\frac{(d(q, p))^{2}}{2}
$$




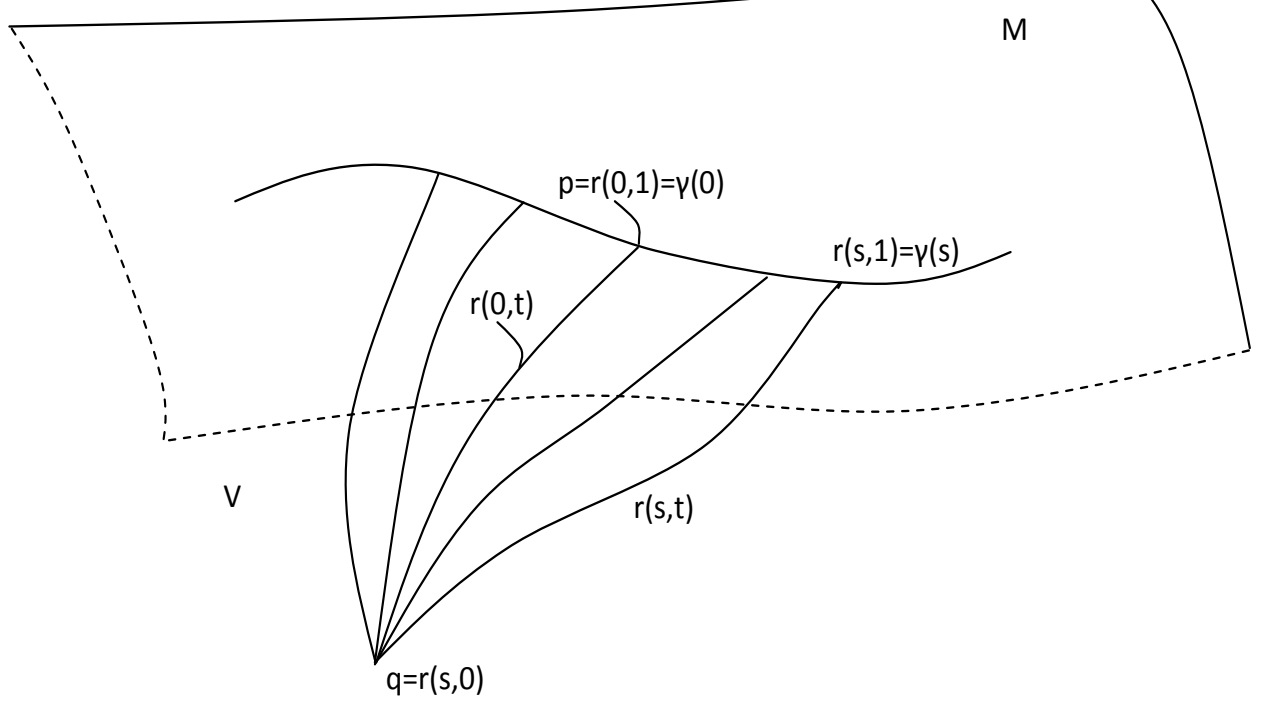

Thus we have

$$
Y(E(p))=d(q, p) Y(d(q, p))
$$

for every $Y \in T M$, and in particular, as $q \notin M$ we have $d(q, p)>0$, then the critical points on $M$ of $E$ are the same of $d(q, \cdot)$. We are going to take the first variation of $E$ along any $Y \in T M$. In order to do that we consider the functional

$$
\begin{gathered}
\varphi:=E \circ \gamma:(-\varepsilon, \varepsilon) \rightarrow \mathbb{R} \\
\varphi(s)=E(\gamma(s))=\frac{(d(q, \gamma(s)))^{2}}{2}=\frac{1}{2} \int_{0}^{1} g(\dot{r}(s, t), \dot{r}(s, t)) d t
\end{gathered}
$$

Therefore

$$
\begin{gathered}
Y(\varphi(s))=\frac{d}{d s} \varphi(s)= \\
=\frac{1}{2} \int_{0}^{1} \frac{d}{d s} g(\dot{r}(s, t), \dot{r}(s, t)) d t=\int_{0}^{1} g\left(\frac{D}{d s} \dot{r}(s, t), \dot{r}(s, t)\right) d t
\end{gathered}
$$

Now

$$
g\left(\frac{D}{d s} \dot{r}(s, t), \dot{r}(s, t)\right)=g\left(\frac{D}{d s} \frac{d}{d t} r(s, t), \dot{r}(s, t)\right)=g\left(\frac{D}{d t} \frac{d}{d s} r(s, t), \dot{r}(s, t)\right)=
$$




$$
=\frac{d}{d t} g\left(\frac{d}{d s} r(s, t), \dot{r}(s, t)\right)-g\left(\frac{d}{d s} r(s, t), \frac{D}{d t} \dot{r}(s, t)\right)=
$$

and

$$
\frac{D}{d t} \dot{r}(s, t)=\nabla_{\dot{r}} \dot{r}=0
$$

therefore by (2) and (3) we have

$$
\begin{aligned}
& Y(\varphi(s))=\int_{0}^{1} \frac{d}{d t} g\left(\frac{d}{d s} r(s, t), \dot{r}(s, t)\right) d t= \\
& =\left[g\left(\frac{d}{d s} r(s, t), \dot{r}(s, t)\right)\right]_{0}^{1}=g\left(Y_{\gamma(s)}, \dot{r}(s, 1)\right)
\end{aligned}
$$

Let us suppose now that $p_{0} \in M$ is a critical point for $E$, then we have $Y(\varphi(0))=0$ for any vector field $Y \in T_{p_{0}} M$ and consequently

$$
\dot{r}(0,1)=-\ell_{\gamma(0)} N=-d\left(q, p_{0}\right) N
$$

Moreover by the assumption (1) we have for every $p \in M$ that

$$
X(\varphi(s))=g\left(X_{\gamma(s)}, \dot{r}(s, 1)\right)=0, \quad \forall s \in(-\varepsilon, \varepsilon)
$$

Now we are going to take the second variation of $E$, twice along $X$, that is: let

$$
\gamma(0)=p, \quad \text { and } \quad \frac{d}{d s} \gamma(s)=X_{\gamma(s)}, \quad \forall s \in(-\varepsilon, \varepsilon)
$$

then we obtain

$$
\begin{aligned}
& 0=X^{2}(\varphi(s))=\frac{d}{d s} g\left(X_{\gamma(s)}, \dot{r}(s, 1)\right)= \\
& =g\left(\frac{D}{d s} X_{\gamma(s)}, \dot{r}(s, 1)\right)+g\left(X_{\gamma(s)}, \frac{D}{d s} \dot{r}(s, 1)\right)= \\
& =g\left(\nabla_{X_{\gamma(s)}} X_{\gamma(s)}, \dot{r}(s, 1)\right)+g\left(X_{\gamma(s)}, \frac{D}{d s} \dot{r}(s, 1)\right)
\end{aligned}
$$


Now if we evaluate the previous expression at a critical value $p_{0}=\gamma(0)$ of $E$, we get:

$$
\begin{aligned}
& 0=g\left(\nabla_{X} X, \dot{r}(0,1)\right)_{p_{0}}+g\left(X_{p_{0}}, \frac{D}{d s} \dot{r}(0,1)\right)= \\
& =-d\left(q, p_{0}\right) g\left(\nabla_{X} X, N\right)_{p_{0}}+g\left(X_{p_{0}}, \frac{D}{d s} \dot{r}(0,1)\right)= \\
& =-d\left(q, p_{0}\right) \mathcal{C}_{p_{0}}^{X}+g\left(X_{p_{0}}, \frac{D}{d s} \dot{r}(0,1)\right)
\end{aligned}
$$

We need to compute

$$
\frac{D}{d s} \dot{r}(0,1)=\left.\frac{D}{d s} \dot{r}(s, t)\right|_{s=0, t=1}=\left.\frac{D}{d s} \frac{d}{d t} r(s, t)\right|_{s=0, t=1}=\left.\frac{D}{d t} \frac{d}{d s} r(s, t)\right|_{s=0, t=1}
$$

Now we explicitly note that

$$
J(t):=\left.\frac{d}{d s} r(s, t)\right|_{s=0}
$$

is a Jacobi field along the geodesic $r(0, \cdot)$, since it is a variation field through geodesics of the geodesic $r(0, \cdot)$. In particular $J$ is normal along $r(0, \cdot)$, in fact:

$$
\begin{gathered}
\frac{d}{d t} g(J(t), \dot{r}(0, t))=\left.\frac{d}{d t} g\left(\frac{d}{d s} r(s, t), \frac{d}{d t} r(s, t)\right)\right|_{s=0}= \\
=\left.g\left(\frac{D}{d t} \frac{d}{d s} r(s, t), \frac{d}{d t} r(s, t)\right)\right|_{s=0}+\left.g\left(\frac{d}{d s} r(s, t), \frac{D}{d t} \frac{d}{d t} r(s, t)\right)\right|_{s=0}= \\
=\left.g\left(\frac{D}{d s} \frac{d}{d t} r(s, t), \frac{d}{d t} r(s, t)\right)\right|_{s=0}=\left.\frac{1}{2} \frac{d}{d s} g\left(\frac{d}{d t} r(s, t), \frac{d}{d t} r(s, t)\right)\right|_{s=0}= \\
=\left.\frac{1}{2} \frac{d}{d s}|\dot{r}(s, t)|^{2}\right|_{s=0}=\left.\frac{1}{2} \frac{d}{d s}\left(\ell_{\gamma(s)}\right)^{2}\right|_{s=0}=0
\end{gathered}
$$

Therefore the function $g(J(t), \dot{r}(0, t))$ is constant along $t$, and for $t=1$ we have:

$$
g(J(1), \dot{r}(0,1))=-d\left(q, p_{0}\right) g\left(X_{p_{0}}, N_{p_{0}}\right)=0
$$


Now we will use the hypothesis on the constant sectional curvature in order to write explicit formulas for the normal Jacobi fields. First we recall that if $K$ is the constant sectional curvature of $V$, then the Riemann curvature endomorphism $R$ satisfies the following identity:

$$
R(X, Y) Z=K(g(Y, Z) X-g(X, Z) Y), \quad \forall X, Y, Z \in T M
$$

Thus, as $J$ satisfies the Jacobi equation, we have

$$
\begin{gathered}
0=\frac{D^{2}}{d t^{2}} J(t)+R(J, \dot{r}(0, t)) \dot{r}(0, t)= \\
=\frac{D^{2}}{d t^{2}} J(t)+K(g(\dot{r}(0, t), \dot{r}(0, t)) J(t)-g(J(t), \dot{r}(0, t)) \dot{r}(0, t))= \\
=\frac{D^{2}}{d t^{2}} J(t)+|\dot{r}(0, t)|^{2} K J(t)=\frac{D^{2}}{d t^{2}} J(t)+\ell_{p_{0}}^{2} K J(t)
\end{gathered}
$$

where $\ell_{p_{0}}=d\left(q, p_{0}\right)$. Now by choosing any parallel vector field $Z$ along the geodesic $r(0, \cdot)$, namely

$$
\frac{D}{d t} Z(t)=\nabla_{\dot{r}(0, t)} Z_{r(0, t)}=0
$$

we can write $J(t)=u(t) Z(t)$, provided the scalar function $u$ satisfies the second order differential equation:

$$
\ddot{u}(t)+\ell_{p_{0}}^{2} K u(t)=0
$$

In particular since we have $J(0)=0$ we require $u(0)=0$ and then we can choose $Z$ such that $Z(1)=X_{p_{0}}$ so that $u(1)=1$. Finally, by setting $a=d\left(q, p_{0}\right) \sqrt{K}$ (for $K \neq 0$ ), we have the explicit formulas:

$$
u(t)= \begin{cases}\frac{1}{\sin (a)} \sin (a t), & K>0 \\ t, & K=0 \\ \frac{1}{\sinh (a)} \sinh (a t), & K<0\end{cases}
$$


We want to note that if $K>0$ then by our hypothesis for every $p \in M$ it holds

$$
0<d(q, p)<\frac{\pi}{\sqrt{K}} \Longrightarrow 0<a<\pi
$$

Now by (5) we have

$$
\frac{D}{d s} \dot{r}(0,1)=\left.\frac{D}{d t} \frac{d}{d s} r(s, t)\right|_{s=0, t=1}=\left.\frac{D}{d t} J(t)\right|_{t=1}=\dot{u}(1) Z(1)=\dot{u}(1) X_{p_{0}}
$$

with

$$
\dot{u}(1)= \begin{cases}a \cot (a), & K>0 \\ 1, & K=0 \\ a \operatorname{coth}(a), & K<0\end{cases}
$$

Therefore (44) rewrites as

$$
0=-d\left(q, p_{0}\right) \mathcal{C}_{p_{0}}^{X}+g\left(X_{p_{0}}, \dot{u}(1) X_{p_{0}}\right)=-d\left(q, p_{0}\right) \mathcal{C}_{p_{0}}^{X}+\dot{u}(1)
$$

Since $X$ is everywhere defined and non-zero, and $M$ is compact, then $E$ admits maximum and minimum which are critical point for $E$. By the hypothesis on the Normal Curvature, $\mathcal{C}_{p}^{X}=\mathcal{C}^{X}=$ const. on $M$, we have that for all the critical points $p_{0}$ of $E$, in particular on the maximum and on the minimum, it holds

$$
\begin{cases}d\left(q, p_{0}\right)=\frac{1}{\sqrt{K}} \arctan \left(\frac{\sqrt{K}}{\mathcal{C}^{X}}\right), & K>0 \\ d\left(q, p_{0}\right)=\frac{1}{\mathcal{C}^{X}}, & K=0 \\ d\left(q, p_{0}\right)=\frac{1}{\sqrt{K}} \operatorname{arctanh}\left(\frac{\sqrt{K}}{\mathcal{C}^{X}}\right), & K<0\end{cases}
$$

Therefore the distance function $d(q, \cdot)$ is constant on $M$ and $M$ is a geodesic sphere. 
Remark 1.4. From the expression of $X^{2}(E(\cdot))$ evaluated at a maximum point $p_{0}$ of the distance function we have in particular

$$
X^{2}\left(E\left(p_{0}\right)\right)=-d\left(q, p_{0}\right) \mathcal{C}_{p_{0}}^{X}+\dot{u}(1) \leq 0
$$

and since $d\left(q, p_{0}\right)$ is strictly positive we have that the constant prescribed for $\mathcal{C}^{X}$ in the hypothesis necessarily satisfies

$$
\mathcal{C}^{X} \geq \frac{\dot{u}(1)}{d\left(q, p_{0}\right)}
$$

\section{Some applications}

We are going to apply the Theorem (1.2) to some manifolds with additional structures. First we treat the case of an almost symplectic manifold with a general symplectic group action, then we will specialize to the case of Kähler manifold with a toric group action; finally we consider the particular case of the Reinhardt domains in $\mathbb{C}^{n+1}$.

Let $V:=V^{2(n+1)}$ be a smooth differentiable manifold of dimension $2(n+1)$. We recall that an almost symplectic structure on $V$ is a 2 -form $\omega$ everywhere non degenerate on $V ; \omega$ is said symplectic if it is close. Moreover an almost complex structure on $V$ is a map $J$ such that for every $p \in M J_{p}$ is a smooth endomorphism on $T_{p} M$ with $J_{p}^{2}=-1 ; J$ is said complex if it is integrable. For every fixed almost symplectic structure $\omega$ there exists and almost complex structure $J$ on $V$ and a compatible metric $g$ on $V$, that means that it holds

$$
\omega(X, Y)=g(X, J Y)
$$

for every pair of vector fields $X, Y \in T V$. Let us consider then any compatible triple $(\omega, J, g)$ on $V$ and a smooth embedded manifold $M$ on $V$, of 
codimension 1. $M$ can always be seen (at least locally) as the (smooth) level set of some function $H$, namely,

$$
H: V \rightarrow \mathbb{R}, \quad M:=\{H=0\}, \quad \nabla H \neq 0 \text { on } M
$$

where $\nabla$ denotes the gradient with respect to $g$. Then it is always possible to define the hamiltonian (non-singular) vector field $X^{H} \in T M$ related to the hamiltonian function $H$ in the following way:

$$
\omega\left(X^{H}, \cdot\right)=-d H(\cdot)
$$

or equivalently, since $d H(\cdot)=g(\nabla H, \cdot)$

$$
X^{H}=J \nabla H
$$

We need to recall some known facts in order to state our next results. First we recall that a symplectic transformation on $V$ is a map $u: V \rightarrow V$ that satisfies $u^{*} \omega=\omega$. A symplectic Lie group action $A$ on $V$ is a group action such that $a$ is a symplectic map on $V$, for every $a \in A$. We have that if the symplectic Lie group action $A$ on $V$ is compact, then there always exists an almost complex structure $J$ on $V$ such that with respect the associated compatible metric $g$ is invariant under the action of $A$ (see for instance [3]): we will call this invariant metric $g_{A}$. We can state now the following corollary of the Theorem (1.2):

Corollary 2.1. Let $(V, \omega)$ an almost symplectic manifold of dimension $2(n+1)$ and let $M$ be a smooth, orientable, compact, connected, with no boundary, embedded manifold on $V$, of codimension 1. Let A a symplectic compact Lie group action on $V$ with a fixed point $q$, such that $M$ is stable under the action of $A$. Suppose $V$ has non-positive constant sectional curvature with respect to the invariant metric $g_{A}$. 
If the Normal Curvature related to the hamiltonian vector field $\mathcal{C}^{X^{H}}$ is constant on $M$, then $M$ is a geodesic sphere with respect to the metric $g_{A}$.

Remark 2.2. For the sake of simplicity we stated the corollary only in the case of non-positive constant sectional curvature: with the additional hypothesis as in Theorem (1.2) (see Remark (1.3)) one can handle the case of positive constant sectional curvature as well.

Proof. First of all, since $A$ is a symplectic group action then $X^{H}$ is one of the vector fields generated by $A$; in particular since $M$ is smooth then $X^{H}$ never vanishes on $M$. Moreover the fixed point $q$ does not belongs to $M$ : in fact if $q \in M$ would mean that all the vector fields generated by the action $A$ vanish at $q$ (by the very definition of fixed point, the orbit of $q$ under the action $A$ is the point $q$ ), and we know that the hamiltonian vector field $X^{H}$ (generated by $A$ ) never vanishes on $M$. Then by considering the distance $d_{A}$ induced by the invariant metric $g_{A}$ on $V$, we have by direct computation that

$$
X^{H}\left(d_{A}(q, p)\right)=0, \quad \forall p \in M
$$

where $q \notin M$ is the fixed point of the action $A$. Then Theorem (1.2) holds.

Now we are going to consider the case of Kähler manifolds. Let $V:=V^{2(n+1)}$ be a smooth differentiable manifold of dimension $2(n+1)$. $V$ is said to be a Kähler manifold if there exists a symplectic structure $\omega$, a complex structure $J$ and a Riemannian metric $g$ such that they are compatible in the sense of (10). We recall that a $2(n+1)$-dimensional symplectic toric manifold is a compact connected symplectic manifold $\left(V^{2(n+1)}, \omega\right)$ equipped with an effective hamiltonian action $A$ of an $n+1$-torus $\mathbb{T}^{(n+1)}$ and with a 
corresponding moment map. We will refer to $V$ as a Kähler toric manifold if in addition the toric action $A$ is holomorphic. We have the following result:

Corollary 2.3. Let $V$ be a symplectic toric Kähler manifold of dimension $2(n+1)$ with non-positive constant sectional curvature and let $M$ be a smooth, orientable, compact, connected, with no boundary, real hypersurface on $V$, stable under the toric group action $A$.

If the Normal Curvature related to the hamiltonian vector field $\mathcal{C}^{X^{H}}$ is constant on $M$, then $M$ is a geodesic sphere.

Proof. As in the previous proof, since $A$ is a symplectic group action then $X^{H}$ is one of the vector fields generated by $A$; moreover since $M$ is smooth then $X^{H}$ never vanishes on $M$. Moreover by the compactness of $V$ and by the results of Atiyah [2], Guillemin-Sternberg [6] and Bredon [5] we have that there exist at least $n+2$ fixed points for the toric group action $A$ and by using the same argument as in the previous proof, we know that none of them is on $M$ : let us choose one and let us call this fixed point $q \notin M$. In addition compatible metric $g$ on this Kähler manifolds is invariant under the group action $A$ : in fact the group action $A$ is holomorphic and then it commutes with the compatible complex structure $J$. As consequence we have that then the following condition is satisfied:

$$
X^{H}\left(d_{g}(q, p)\right)=0, \quad \forall p \in M
$$

where $d_{g}$ is the distance function induced by the invariant metric $g$. Then Theorem (1.2) holds.

Now we will consider $M$ as the smooth boundary of a Reinhardt domain in $\mathbb{C}^{n+1}$. A Reinhardt domain $\Omega$ (with center at the origin) is by definition an 
open subset of $\mathbb{C}^{n+1}$ such that

$$
\text { if }\left(z_{1}, \ldots, z_{n+1}\right) \in \Omega \quad \text { then } \quad\left(e^{i \theta_{1}} z_{1}, \ldots, e^{i \theta_{n+1}} z_{n+1}\right) \in \Omega
$$

for all the real numbers $\theta_{1}, \ldots, \theta_{n+1}$. These domains naturally arise in the theory of several complex variables as the logarithmically convex Reinhardt domains are the domains of convergence of power series (see for instance [7, [10]). The smooth boundary $M:=\partial \Omega$ is then a smooth real hypersurface in $\mathbb{C}^{n+1}$ and thus a CR-manifold of CR-codimension equal to one, with the standard CR structure induced by the holomorphic structure of $\mathbb{C}^{n+1}$. Thus for every $p \in M$ the tangent space $T_{p} M$ splits in two subspaces: the $2 n$-dimensional horizontal subspace $H_{p} M$, the largest subspace in $T_{p} M$ invariant under the action of the standard complex structure $J$ of $\mathbb{C}^{n+1}$ and the vertical one-dimensional subspace generated by the characteristic direction $T_{p}:=J N_{p}$, where $N_{p}$ is the unit normal at $p$. Moreover, if $g$ is the standard metric on $\mathbb{C}^{n+1}$, then it holds

$$
T_{p} M=H_{p} M \oplus \mathbb{R} T_{p}
$$

and the sum is $g$-orthogonal.

Let $\nabla$ be the Levi-Civita connection for $g$ and let us consider the complexified horizontal space

$$
H^{\mathbb{C}} M:=\{Z=X-i J \cdot X: X \in H M\}
$$

The Levi Form $l$ is then the sesquilinear and hermitian operator on $H^{\mathbb{C}} M$ defined in the following way: $\forall Z_{1}, Z_{2} \in H^{\mathbb{C}} M$

$$
l\left(Z_{1}, Z_{2}\right)=\widetilde{g}\left(\widetilde{\nabla}_{Z_{1}} \bar{Z}_{2}, N\right)
$$


One then compares the Levi Form with the Second Fundamental Form $h$ of $M$ by using the identity [4]

$$
l(Z, Z)=h(X, X)+h(J(X), J(X)), \quad \forall X \in H M
$$

Definition 2.4. We will call $\mathcal{C}^{T}=h(T, T)=g\left(\nabla_{T} T, N\right)$ the Characteristic Curvature of $M$.

Thus, a direct calculation leads to the relation between the classical Mean Curvature $H$, the Levi-Mean Curvature $L$ and the Characteristic Curvature $\mathcal{C}^{T}$ of $M$ :

$$
H=\frac{1}{2 n+1}\left(2 n L+\mathcal{C}^{T}\right)
$$

Following a couple of papers by Hounie and Lanconelli ([8] , 9]) in which they prove Alexandrov type theorems for Reinhardt domains in $\mathbb{C}^{n+1}$ using the Levi Mean Curvature, the second author in [11] proved a similar symmetry result for Reinhardt domains in $\mathbb{C}^{n+1}$ starting from the Characteristic Curvature rather than the Levi ones:

Theorem 2.5. Let $M:=\partial \Omega$ be the smooth boundary of a bounded Reinhardt domain $\Omega$ in $\mathbb{C}^{n+1}$. If the characteristic curvature $\mathcal{C}^{T}$ is constant then $M$ is a sphere of radius equal to $1 / \mathcal{C}^{T}$.

Here we show that this result is a corollary of our main Theorem (1.2)

Proof. We can think of $\mathbb{C}^{n+1}$ as a Kähler manifold with the standard compatible symplectic, complex and metric structures and with sectional curvature identically zero. We recall now that for every hypersurface $M$ in $\mathbb{C}^{n+1}$, with $f$ as defining function, the characteristic direction $T$ of $M$ is exactly the (normalized) hamiltonian vector field for the hamiltonian function $f$. Moreover by the very definition of Reinhardt domain (13) we recognize that 
there exist an explicit toric group action $A$ on $\mathbb{C}^{n+1}$ such that $M$ is stable

under $A$. Since $\mathbb{C}^{n+1}$ is non compact we note that we do not have a symplectic toric Kähler manifold, but in this particular situation we have that the origin is a fixed point for $A$ and it does not belong to $M$. Then Theorem (1.2) holds.

\section{References}

[1] A.D. Alexandrov, A characteristic property of spheres, Ann. Mat. Pura Appl., (4) 58, pag. 303-315, 1962

[2] M. АтіYAн, Convexity and commuting Hamiltonians, Bull. London Math. Soc.14 (1982), 1-15.

[3] M. Audin, Torus Actions on Symplectic Manifolds. Second revised edition. Progress in Mathematics, 93. Birkhuser Verlag, Basel, 2004.

[4] A. Bogges, CR Manifolds and the Tangential Cauchy-Riemann Complex, Studies in Advanced Mathematics, 1991

[5] G. Bredon, Introduction to Compact Transformation Groups, Pure and Applied Mathematics 46, Academic Press, New York-London, 1972.

[6] V. Guillemin, S. Sternberg, Convexity properties of the moment mapping, Invent. Math. 67 (1982), 491-513.

[7] L. HöRmander, An introduction to complex analysis in several variables, North-Holland (1973) 
[8] J.G. Hounie, E. Lanconelli, An Alexander type Theorem for Reinhardt domains of $\mathbb{C}^{2}$. Recent progress on some problems in several complex variables and partial differential equations, Contemp. Math., Amer. Math. Soc., Providence, RI, 400,129-146, 2006.

[9] J.G. Hounie, E. Lanconelli, A sphere theorem for a class of Reinhardt domains with constant Levi curvature, Forum Mathematicum $200820: 4,571-586$

[10] M. JARnicki, P. Pflug, First steps in several complex variables: Reinhardt domains, European Mathematical Society, 2008

[11] V. Martino, A symmetry result on Reinhardt domains, submitted 